\title{
Switching chiral solitons for algebraic operation of topological quaternary digits
}

\author{
Tae-Hwan Kim ${ }^{1 \star}$, Sangmo Cheon ${ }^{2}$ and Han Woong Yeom ${ }^{1,2 \star}$
}

\begin{abstract}
Chiral objects can be found throughout nature ${ }^{1-4} ;$ in condensed matter chiral objects are often excited states protected by a system's topology. The use of chiral topological excitations to carry information has been demonstrated, where the information is robust against external perturbations $\mathbf{s}^{5,6}$. For instance, reading, writing, and transfer of binary information have been demonstrated with chiral topological excitations in magnetic systems, skyrmions ${ }^{7-14}$, for spintronic devices ${ }^{13-19}$. The next step is logic or algebraic operations of such topological bits ${ }^{20-22}$. Here, we show experimentally the switching between chiral topological excitations or chiral solitons of different chirality in a one-dimensional electronic system with $Z_{4}$ topological symmetry ${ }^{23,24}$. We found that a fast-moving achiral soliton merges with chiral solitons to switch their handedness. This can lead to the realization of algebraic operation of $Z_{4}$ topological charges ${ }^{25}$. Chiral solitons could be a platform for storage and operation of robust topological multi-digit information.
\end{abstract}

Topological particle-like excitations can be used as information carriers in next-generation non-volatile memory and logic devices on the basis of their non-perturbative topological robustness. Promising candidates for topological information carriers using such topological excitations have been discussed actively in magnetic systems-for example, domain walls for racetrack memory devices $^{5,6}$ and skyrmions ${ }^{7-14}$, two-dimensional chiral spin configurations protected topologically ${ }^{13-19}$. However, neighbouring domain walls with opposite chirality would annihilate each other under the influence of an applied field in the former case. On the other hand, ironically, the chirality of skyrmions is very difficult to switch due to the intrinsic parity-breaking chiral interaction ${ }^{6,26}$. For these reasons, although binary-digit memory devices based on skyrmions have been successful demonstrated, the realization of logic operations with magnetic topological excitations faces huge obstacles requiring sophisticated nanoscale fabrication and precise material engineering ${ }^{20-22}$.

On the other hand, topological excitations have been known for a long time in one-dimensional electronic systems; a Peierlsdistorted atomic chain such as polyacetylene has two topologically distinct ground states, which are connected by a unique topological $\operatorname{soliton}^{27-31}$. This topological excitation does not have such chirality $^{24}$ as magnetic solitons ${ }^{32-34}$ and skyrmions ${ }^{7-14}$. However, our team recently discovered new kinds of topological solitons having chirality in double Peierls chains of indium atomic wires ${ }^{23,24}$. Double Peierls chains uniquely have topologically distinct four-fold degenerate ground states: the topology of the energetically degenerate ground states is distinguished in the topological order-parameter space using the order parameters $m_{x}$ and $m_{z}$, representing sublattice dimerization and interchain coupling potential terms induced by the spontaneous symmetry breaking, respectively ${ }^{24}$. The four-fold ground states are denoted as $\mathrm{Aa}, \mathrm{Ab}, \mathrm{Ba}$ and $\mathrm{Bb}$ for each Peierls chain (see Fig. 1a and Supplementary Fig. 1). These ground states can be connected by three different topological excitations or solitons (Fig. 1a): two chiral states with different handedness (Fig. 1b,c) and one state without chirality (Fig. 1d). Such solitons including a null state can carry quaternary-digit information in terms of topological charges. Furthermore, due to their unique four-fold degeneracy (or the $Z_{4}$ symmetry), $Z_{4}$ algebraic operations would be possible in principle among these solitons and a null state, as demonstrated below. Thus, these topological chiral solitons can be utilized not only for multi-digit memories in electronic systems, but also for logic devices using topological excitations. In contrast to the logic operation proposed with skyrmions, the chiral soliton operation in this work does not need sophisticated engineering since it is intrinsic to the system's $Z_{4}$ symmetry.

The chirality of solitons is distinguished by their distinct structures and electronic states ${ }^{24}$. In a wire of double indium zigzag chains, a topological soliton can exist on either of the two chains by connecting two degenerate Peierls-dimerized states on a single chain. The soliton in one chain has to interact with the bonding or anti-bonding orbital of the neighbouring chain (Fig. 1b,c). This interaction changes the sublattice parity and provides the chirality to the soliton ${ }^{24}$ : right-chiral (RC) and left-chiral (LC) solitons result from the interaction with bonding (Fig. 1b) and anti-bonding orbitals (Fig. 1c) of the neighbouring chain, respectively. RC and LC solitons are distinct in scanning tunnelling microscopy (STM) images, as shown in Fig. 1e,f. On the other hand, when both chains have solitonic phase boundaries at the same time, a third type of a soliton without chirality emerges, which will be called achiral (AC) soliton hereafter (Fig. 1d,g). These three types of solitons constitute a complete set of a $Z_{4}$ topological system with four-fold degenerate ground states of double Peierls-distorted chains. The distinction of different types of solitons is more striking in their electronic states within the charge-density-wave bandgap. The electronic states of RC (LC) solitons emerge below (above) the Fermi level within the bandgap, whereas AC solitons have their states both above and below the Fermi level (Supplementary Fig. 2a ${ }^{23,24}$. The electronic difference results in the fact that RC solitons always looks brighter in STM images than LC solitons due to their enhanced density of states in filled states (Supplementary Fig. 2b-d).

Topological solitons are highly mobile, but are occasionally trapped by structural defects or impurities to allow the STM observation $^{23}$. Spontaneously charged solitons ${ }^{24}$ would be easily trapped by a defect charged oppositely through the influence of a Coulomb attraction. The Coulomb interaction would shrink the size of a trapped soliton significantly $\mathrm{y}^{30}$. In Fig. $1 \mathrm{~h}$, a LC soliton is trapped by two defects (indicated by $\mathrm{D}_{1}, \mathrm{D}_{2}$ ), which connect the same ground states as LC solitons with a much shorter characteristic length

${ }^{1}$ Department of Physics, Pohang University of Science and Technology (POSTECH), Pohang 37673, Korea. ${ }^{2}$ Center for Artificial Low Dimensional Electronic Systems, Institute for Basic Science (IBS), Pohang 37673, Korea. *e-mail: taehwan@postech.ac.kr; yeom@postech.ac.kr 

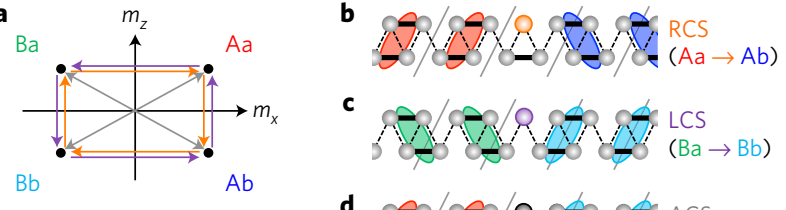

d
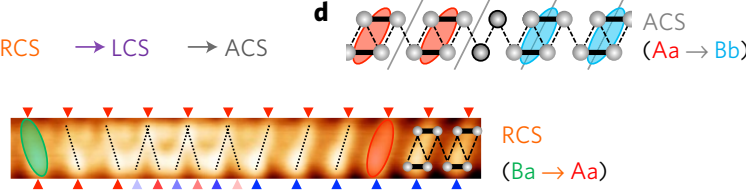

$\mathbf{f}$

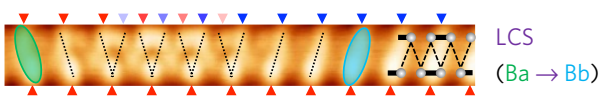

g
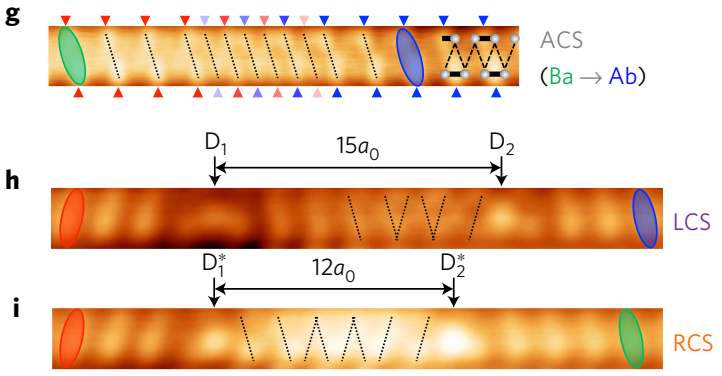

$\mathbf{j}$

$\mathbf{k}$

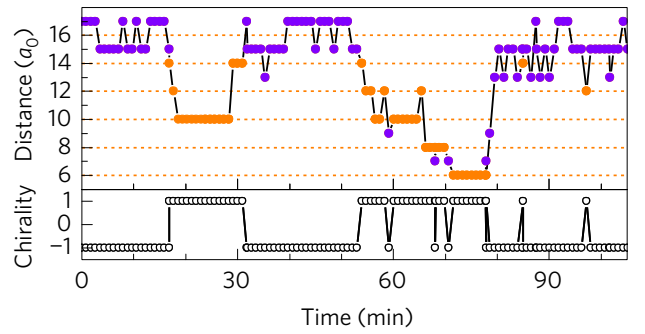

Figure 1 | Characterizing chiral switching between topological solitons.

a, Four-fold ground states of a lower Peierls-distorted chain in the order-parameter space denoted by $\mathrm{Aa}, \mathrm{Ab}, \mathrm{Ba}$ and $\mathrm{Bb}$ (see Supplementary Fig. 1). Arrows connecting two ground states indicate right-chiral (RC), left-chiral (LC) and achiral (AC) solitons. b-d, Schematics of RC (b), LC (c) and $A C$ (d) solitons. Coloured ovals represent four-fold degenerate ground states. For simplicity, the size of solitons is shown reduced. Thick solid and dashed lines between atoms indicate Peierls dimers and interchain coupling, respectively. e-g, STM images showing unbound RC (e), LC (f) and AC (g) solitons. Red and blue triangles as well as dotted lines illustrate the phase shift on solitons. $\mathbf{h}, \mathbf{i}$, Sequential STM imaging reveals chiral switching between $\mathrm{LC}\left(\mathbf{h}, T=t_{0}\right)$ and $\mathrm{RC}\left(\mathbf{i}, T=t_{1}\right)$ solitons. The distance between two trapping defects (indicated by $D_{1}, D_{2}$ or $D_{1}^{*}, D_{2}^{*}$ ) holding chiral solitons is directly related to the chirality of the soliton between them. The ground state on the right-hand side of the $D_{2}$ defect is also altered according to the chirality. Dotted lines indicate the phase shift on solitons. j, Time-dependent distance between two defects trapping the chiral soliton drawn from consecutive STM imaging. $\mathbf{k}$, Time-dependent chirality obtained from the polarity of the defect distance as well as STM images.

(see Supplementary Fig. 3) $)^{35,36}$. The trapping defect $\left(D_{2}\right)$ makes the LC soliton shorter (Fig. 1h) than one without defects (Fig. 1f), as expected from the attractive Coulomb interaction between solitons and trapping defects (see further discussion below). Note that the characteristic electronic state of a soliton ${ }^{24}$ remains intact upon trapping, thanks to its non-perturbative topological robustness, as demonstrated below.

During long-term STM observations of a trapped chiral soliton (Fig. 1h,i and Supplementary Fig. 4), its handedness was found to change abruptly and occasionally (Fig. 1j,k). To understand the mechanism of the chiral switching of solitons, we note the fact that each chiral switching is accompanied by the hopping of a trapping defect. As shown in Fig. $1 \mathrm{j}$, one of the two trapping defects $\left(D_{2}\right)$ hops along the chain by an integer multiple of the lattice unit cell $\left(a_{0}=0.384 \mathrm{~nm}\right)$. Figure $1 \mathrm{j}, \mathrm{k}$ indicates with no doubt that this defect hopping is uniquely related to the handedness of chiral solitons in between the trapping defects. That is, an odd- $a_{0}$-hopping always changes the chirality of the trapped soliton whereas an even- $a_{0}$ hopping does not. Since the periodicity of the dimerized Peierls chain is $2 a_{0}$, the odd- $a_{0}$-hopping of a trapping defect inevitably shifts nearby ground states from one to another, in contrast to the even$a_{0}$-hopping maintaining the same ground states before/after the hopping (see Supplementary Fig. 4). Thus the odd- $a_{0}$-hopping must involve another soliton. Indeed, our previous work established that the odd- $a_{0}$-hopping is induced by an AC soliton passing through the trapping defects ${ }^{23}$. Therefore, we can suspect that a fast-moving AC soliton giving rise to the odd- $a_{0}$-hopping is related to switching the handedness of a chiral soliton.

This occasional but very fast switching is not fully captured by repeated STM imaging with a scan interval of $50 \mathrm{~s}$, as shown in Fig. 1j. However, a scanning tunnelling spectroscopy (STS) measurement at a fixed location repeated every $3 \mathrm{~s}$ can track switching events better due to the unique electronic states of AC, RC and LC solitons (Supplementary Fig. 2a $)^{24}$. The temporal resolution for an event changing a particular electronic state can be as fast as $0.01 \mathrm{~s}$ in this measurement, $\sim 5,000$ times faster than that of sequential STM imaging. As shown in Supplementary Fig. 5 and Fig. 2b, for a particular series of four sequential STS voltage sweeps on the trapped soliton, the density of states (the intensity of the normalized $\mathrm{d} I / \mathrm{d} V$ signal, where $I$ and $V$ are the tunnelling current and bias voltage across the sample with respect to the tip) changes abruptly twice (indicated by red arrows). By comparing the STS spectra before and after these events with the reference spectra of chiral solitons, the initial LC soliton is found to switch to the RC soliton $(t=3.1 \mathrm{~s})$ and back to the LC soliton $(t=5.4 \mathrm{~s})$ (Supplementary Fig. 5). For an extended STS observation on a single chiral soliton, we can observe many telegraphlike switchings between the states above and below the Fermi level (Fig. 2a,c). Each switching in electronic states can be determined as the chiral switching of the soliton (Fig. 2d), since the in-gap states have one-to-one correspondence with the chirality of solitons (Supplementary Fig. 2a). In contrast to the chiral switching, one can find a high STS intensity for both filled and empty states at the same time, leading to almost zero chiral asymmetry (marked with the red oval in Fig. 2d), which corresponds to the STS spectra of AC solitons (the inset of Fig. $2 \mathrm{~d})^{24}$. This finding indicates unambiguously that AC solitons momentarily exist as required for the odd- $a_{0}$-hopping of trapping defects and switching the handedness of chiral solitons.

We show further below that the AC soliton is indeed topologically required to switch the chirality of the soliton. Figure 3 a shows the schematic diagrams describing the chiral switching of a LC soliton induced by a left-going AC soliton. For simplicity, we ignore the trapping defects (see Fig. 3b when including the trapping defects). Initially, both the LC and AC solitons are away from each other while bridging different ground states. When they come close enough to each other, the ground state between two solitons disappears and the two solitons together become equivalent to a RC soliton due to the given boundary condition. Thus, they can eventually merge to create a RC soliton as a result of energetic considerations: one soliton has a lower energy than two solitons (Fig. 3c and Supplementary Fig. 6). More detailed energetics will be considered below. If another AC soliton approaches, the RC soliton would switch back to a LC soliton through the same procedure. This consideration does not change in the case of trapped solitons in between two defects, as shown in Fig. $3 b$.

This chiral switching is in fact intrinsic to the $Z_{4}$ topology of the present system. For the particular chiral switching in Fig. 3a, $\mathrm{a} \mathrm{Ba} \rightarrow \mathrm{Bb}(\mathrm{LC})$ soliton and $\mathrm{ab} \rightarrow \mathrm{Aa}(\mathrm{AC})$ soliton merge into 

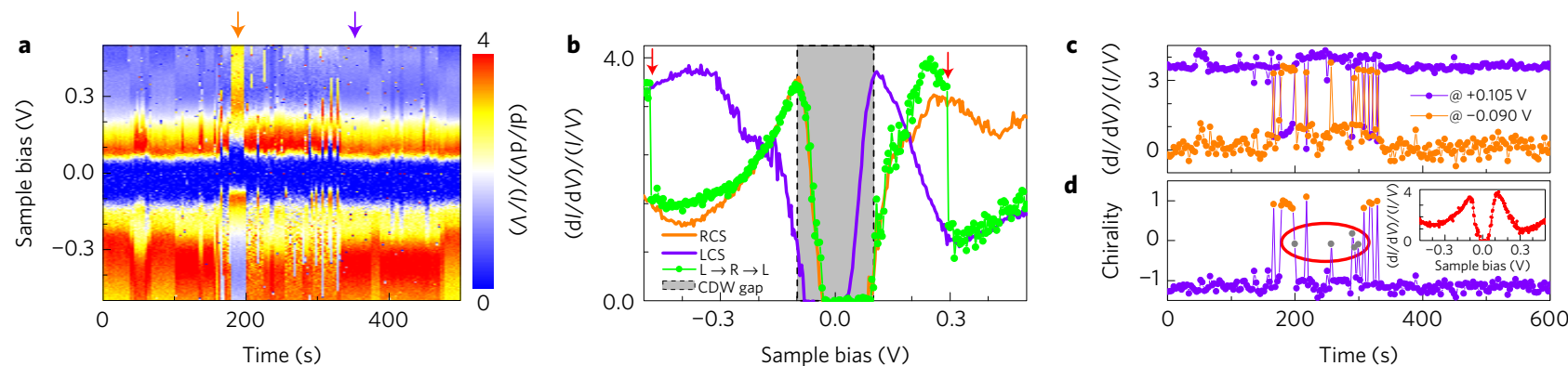

Figure $\mathbf{2}$ | Trace section of the time-dependent chiral switching. a, Time-traced STS spectra clearly show two dominant soliton states indicated by orange (RC) and purple (LC) arrows as well as several abrupt switching events between LC and RC soliton states. $\mathbf{b}$, The green curve (see Supplementary Fig. 5) shows chiral switching events from LC to RC and then from RC to LC indicated by red arrows. The charge-density-wave gap is highlighted by the shaded regions. c, Time-dependent $\mathrm{d} / \mathrm{d} \mathrm{d}$ signal of soliton states collected at $+0.105 \mathrm{~V}$ (purple dots for $\mathrm{LC}$ soliton states, $I_{\mathrm{L}}$ ) and $-0.090 \mathrm{~V}$ (orange dots for $\mathrm{RC}$ soliton states, $\left.I_{R}\right)$ from $\mathbf{a}$. d, Time-dependent chirality defined by asymmetry of $d / / d V$ signals, $A=\left(I_{R}-I_{L}\right) /\left(I_{R}+I_{L}\right)$. A red oval indicates intermediate states between LC and RC. Inset shows the STS spectrum obtained at one of the intermediate states, which clearly identifies an AC soliton.
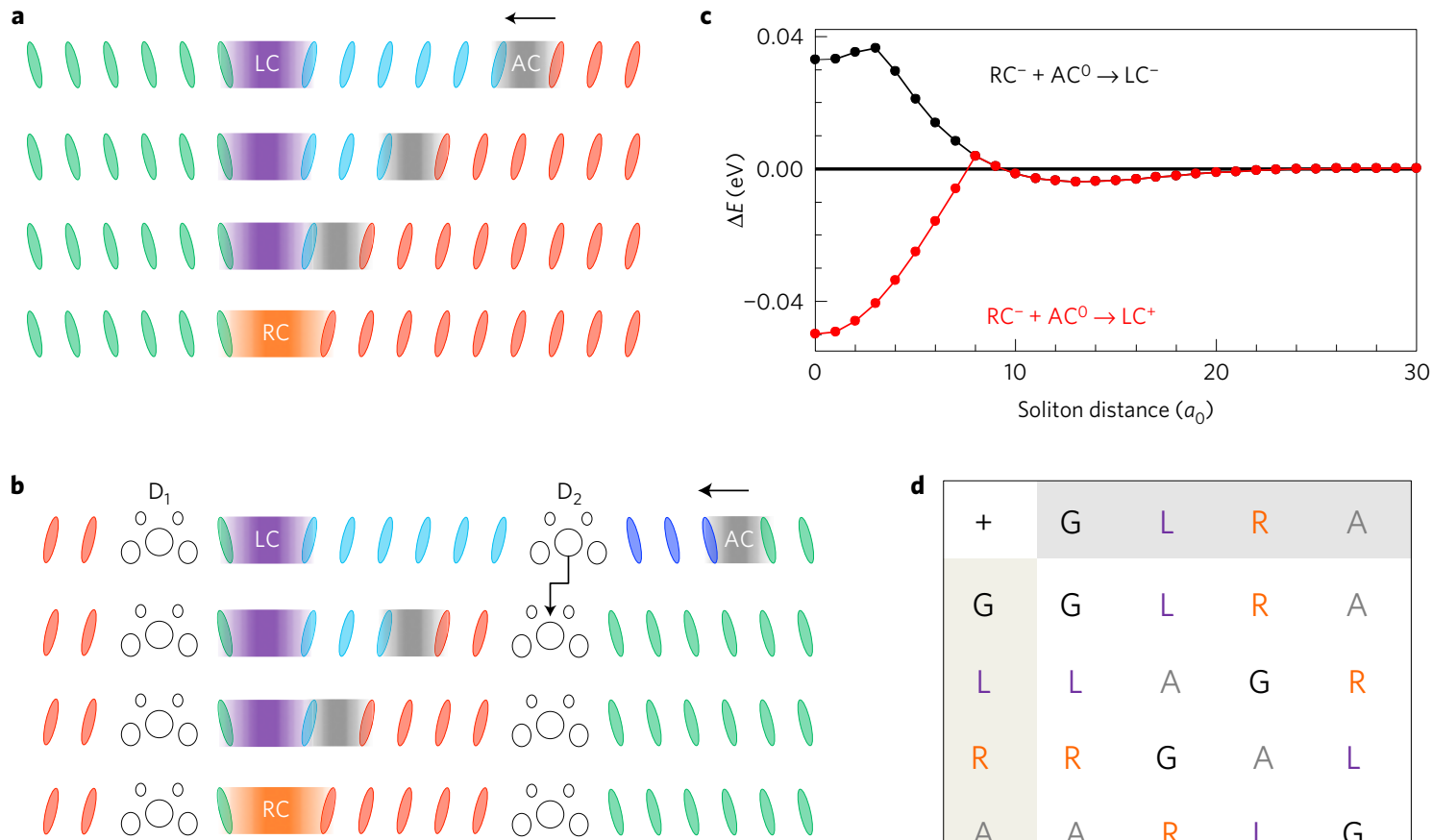

\begin{tabular}{|ccccc}
+ d & $G$ & $L$ & $R$ & $A$ \\
$G$ & $G$ & $L$ & $R$ & $A$ \\
$L$ & $L$ & $A$ & $G$ & $R$ \\
$R$ & $R$ & $G$ & $A$ & $L$ \\
$A$ & $A$ & $R$ & $L$ & $G$ \\
\hline
\end{tabular}

Figure $\mathbf{3}$ | Algebraic operation using solitons. a,b, Schematics showing chiral switching from LC to RC by an AC soliton without (a) and with (b) the defects indicated by $D_{1}$ or $D_{2}$. Coloured ovals represent four-fold degenerate ground states. $\mathbf{c}$, Total energy change as a function of the distance between RC and AC. A red (black) curve indicates the soliton merging with (without) the charge transfer from/to trapping defects. d, Algebraic operation table showing a $Z_{4}$ abelian group. $G, A, L$ and $R$ indicate ground states, $A C, L C$ and $R C$ solitons, respectively.

$\mathrm{a} \mathrm{Ba} \rightarrow \mathrm{Aa}(\mathrm{RC})$ soliton. In another case, two LC solitons merge into an $\mathrm{AC}$ soliton: $\mathrm{Aa} \rightarrow \mathrm{Ba}+\mathrm{Ba} \rightarrow \mathrm{Bb}=\mathrm{Aa} \rightarrow \mathrm{Bb}$ (Figs 1a and $3 \mathrm{~d}$ ). We can expand this consideration to the cyclic group under addition modulo four with four elements consisting of LC, RC and AC solitons as well as an identity element (denoted as G in Fig. 3d, indicating the ground state without a soliton). This cyclic group of the present system is the same as a $Z_{4}$ abelian four-element group ${ }^{25}$ and the soliton switching corresponds to the algebraic operation of $Z_{4}$ topological charges (Supplementary Fig. 7).

We can elaborate the energetics of the merging of two solitons. Self-consistent tight-binding calculations based on the generalized $\mathrm{Su}-\mathrm{Sch}$ rieffer-Heeger Hamiltonian (see ref. 24 and its Supplementary Methods) are utilized to estimate the soliton formation energies. Neutral AC solitons are the lowest topological excitations, whereas LC and RC solitons prefer to be positively and negatively charged, respectively, when they are created (see Supplementary Fig. 6). In the case of the $\mathrm{RC}+\mathrm{AC} \rightarrow \mathrm{LC}$ operation, a negatively charged RC soliton and a neutral AC soliton should merge into a negatively charged LC soliton according to charge conservation. However, since a negatively charged LC soliton has a much higher formation energy, the charge-conserved merging is energetically unfavourable (black curve in Fig. 3c). Only when the merged LC soliton becomes positively charged, possibly through charge transfer from/to nearby trapping defects, a merged soliton can have an energy gain over the energy sum of the two initial solitons (red curve in Fig. 3c). This charge transfer from/to the trapping defects is not directly probed, but is plausible since the Coulomb interaction between charged solitons and trapping defects is a prerequisite for the soliton trapping observed. The charge transfer is further indicated by the observation that the apparent STM heights of defects systemically change depending on the chirality of the solitons (Supplementary Fig. 8).

In addition to the Coulomb interaction, a chiral soliton itself can modify the strain field near trapping defects, which may be 
another driving mechanism of binding between chiral solitons and trapping defects. We tried to analyse the motion of the trapping defects (Fig. 1j and Supplementary Fig. 4d) with a simple onedimensional random walk theory ${ }^{37}$. As shown in Supplementary Fig. 9, the trapping defects show biased or unbiased random walk for a short period of time, but eventually deviate from the random walk. This strongly indicates two trapping defects prefer being close to each other, due probably to the strain field of solitons in between the trapping defects.

To realize logic devices utilizing chiral soliton, one needs to selectively control the creation and/or motion of the topological solitons. The trapping defects $\left(D_{1}, D_{2}\right.$ in Fig. 1$)$ can play a crucial role in creating various solitons, as proposed in Supplementary Fig. 10. If one creates a defect by using atom manipulation ${ }^{38}$, the emerging defect should be compensated by creating a RC soliton to preserve an initial unperturbed boundary condition (Supplementary Fig. 10a,b). By adding defects sequentially, one can change from $\mathrm{RC}$ to $\mathrm{AC}$, from $\mathrm{AC}$ to $\mathrm{LC}$, and from $\mathrm{LC}$ to the ground state (Supplementary Fig. 10). In addition, the preliminary experimental observation suggests that specific end configurations of chains can generate AC solitons very frequently (Supplementary Fig. 11). Therefore, a controllable logic device based on chiral solitons is in principle possible.

We have demonstrated that two solitons with different chirality can merge into another chiral soliton. For typical solitons, and most of other topological excitations observed, the algebraic operations are rather trivial, such as annihilation of two excitations due to their simpler $Z_{2}$ topology. The unprecedented non-trivial operation of topological excitations yielding a different soliton is realized because of the unique $Z_{4}$ topology. The switching between three different types of solitons allows us to use them as quaternary-digit information carriers as well as logic operators. This will open a door towards logic devices using chiral topological excitations, and more generally into a new field of electronics called 'solitonics'.

\section{Methods}

Methods, including statements of data availability and any associated accession codes and references, are available in the online version of this paper.

\section{Received 19 April 2016; accepted 3 January 2017;} published online 6 February 2017

\section{References}

1. Simon, J. Magnetic fields without magnetic fields. Nature 515, 202-203 (2014).

2. Shelke, S. A. \& Piccirilli, J. A. RNA made in its own mirror image. Nature 515, 347-348 (2014)

3. Romanov-Michailidis, F. \& Rovis, T. Natural polarity inverted. Nature 523, 417-418 (2015).

4. Boyd, R. W. Neutrons with a twist. Nature 525, 462-464 (2015).

5. Parkin, S. S. P., Hayashi, M. \& Thomas, L. Magnetic domain-wall racetrack memory. Science 320, 190-194 (2008).

6. Braun, H.-B. Topological effects in nanomagnetism: from superparamagnetism to chiral quantum solitons. Adv. Phys. 61, 1-116 (2012).

7. Bode, M. et al. Chiral magnetic order at surfaces driven by inversion asymmetry. Nature 447, 190-193 (2007).

8. Mühlbauer, S. et al. Skyrmion lattice in a chiral magnet. Science 323, 915-919 (2009).

9. Heinze, S. et al. Spontaneous atomic-scale magnetic skyrmion lattice in two dimensions. Nat. Phys. 7, 713-718 (2011).

10. Yu, X. Z. et al. Near room-temperature formation of a skyrmion crystal in thin-films of the helimagnet FeGe. Nat. Mater. 10, 106-109 (2011).

11. Milde, P. et al. Unwinding of a skyrmion lattice by magnetic monopoles. Science 340, 1076-1080 (2013).

12. Nagaosa, N. \& Tokura, Y. Topological properties and dynamics of magnetic skyrmions. Nat. Nanotech. 8, 899-911 (2013).

13. Romming, N. et al. Writing and deleting single magnetic skyrmions. Science 341, 636-639 (2013).
14. Hagemeister, J., Romming, N., von Bergmann, K., Vedmedenko, E. Y. \& Wiesendanger, R. Stability of single skyrmionic bits. Nat. Commun. 6, 8455 (2015).

15. Fert, A., Cros, V. \& Sampaio, J. Skyrmions on the track. Nat. Nanotech. 8, 152-156 (2013)

16. Sampaio, J., Cros, V., Rohart, S., Thiaville, A. \& Fert, A. Nucleation, stability and current-induced motion of isolated magnetic skyrmions in nanostructures. Nat. Nanotech. 8, 839-844 (2013).

17. Hanneken, C. et al. Electrical detection of magnetic skyrmions by tunnelling non-collinear magnetoresistance. Nat. Nanotech. 10, 1039-1042 (2015).

18. Jiang, W. et al. Blowing magnetic skyrmion bubbles. Science $\mathbf{3 4 9}$, 283-286 (2015)

19. Zhang, X., Zhou, Y., Ezawa, M., Zhao, G. P. \& Zhao, W. Magnetic skyrmion transistor: skyrmion motion in a voltage-gated nanotrack. Sci. Rep. 5, 11369 (2015).

20. Zhang, S., Baker, A. A., Komineas, S. \& Hesjedal, T. Topological computation based on direct magnetic logic communication. Sci. Rep. 5, 15773 (2015).

21. Zhang, X. et al. All-magnetic control of skyrmions in nanowires by a spin wave. Nanotechnology 26, 225701 (2015).

22. Zhang, X., Ezawa, M. \& Zhou, Y. Magnetic skyrmion logic gates: conversion, duplication and merging of skyrmions. Sci. Rep. 5, 9400 (2015).

23. Kim, T.-H. \& Yeom, H. W. Topological Solitons versus nonsolitonic phase defects in a quasi-one-dimensional charge-density wave. Phys. Rev. Lett. 109, 246802 (2012).

24. Cheon, S., Kim, T.-H., Lee, S.-H. \& Yeom, H. W. Chiral solitons in a coupled double Peierls chain. Science 350, 182-185 (2015).

25. Lovett, S. Abstract Algebra: Structures and Applications 87-88 (CRC Press, 2015)

26. Dzyaloshinsky, I. A thermodynamic theory of 'weak' ferromagnetism of antiferromagnetics. J. Phys. Chem. Solids 4, 241-255 (1958).

27. Jackiw, R. \& Rebbi, C. Solitons with fermion number $1 / 2$. Phys. Rev. D 13, 3398-3409 (1976).

28. Su, W. P., Schrieffer, J. R. \& Heeger, A. J. Solitons in polyacetylene. Phys. Rev. Lett. 42, 1698-1701 (1979).

29. Brazovskii, S. A., Gordynin, S. A. \& Kirova, N. N. Exact solution of the Peierls model with an arbitrary number of electrons in the unit cell. Pis'ma $v$ Zh. Eksp. Teor. Fiz. 31, 486-491 (1980).

30. Su, W. P., Schrieffer, J. R. \& Heeger, A. J. Soliton excitations in polyacetylene. Phys. Rev. B 22, 2099-2111 (1980).

31. Vanderbilt, D. \& Mele, E. J. Effects of disorder on the electronic structure of undoped polyacetylene. Phys. Rev. B 22, 3939-3948 (1980).

32. Braun, H.-B. et al. Emergence of soliton chirality in a quantum antiferromagnet. Nat. Phys. 1, 159-163 (2005).

33. Togawa, Y. et al. Chiral magnetic soliton lattice on a chiral helimagnet. Phys. Rev. Lett. 108, 107202 (2012)

34. Togawa, Y. et al. Magnetic soliton confinement and discretization effects arising from macroscopic coherence in a chiral spin soliton lattice. Phys. Rev. B 92, 220412 (2015).

35. Zhang, H. et al. Atomic structure, energetics, and dynamics of topological solitons in indium chains on $\mathrm{Si}(111)$ surfaces. Phys. Rev. Lett. 106, 026801 (2011).

36. Yeom, H. W., Oh, D. M., Wippermann, S. \& Schmidt, W. G. Impurity-mediated early condensation of a charge density wave in an atomic wire array. ACS Nano 10, 810-814 (2016).

37. Senft, D. C. \& Ehrlich, G. Long jumps in surface diffusion: one-dimensional migration of isolated adatoms. Phys. Rev. Lett. 74, 294-297 (1995).

38. Hla, S. W. Atom-by-atom assembly. Rep. Prog. Phys. 77, 056502 (2014).

\section{Acknowledgements}

We thank S.-H. Lee for discussions in the early stages and technical help on the calculation method. This work was supported by IBS-R014-D1. T.-H.K. was supported by Basic Science Research Program (Grant No. NRF-2014R1A1A1002205) and the SRC Center for Topological Matter (Grant No. 2011-0030046) through the National Research Foundation (NRF) of Korea funded by the Ministry of Science, ICT \& Future Planning.

\section{Author contributions}

T.-H.K. performed STM/STS measurements; S.C. performed tight-binding calculations; T.-H.K. and H.W.Y. analysed data and wrote the paper. All authors discussed the results and commented on the manuscript.

\section{Additional information}

Supplementary information is available in the online version of the paper. Reprints and permissions information is available online at www.nature.com/reprints.

Correspondence and requests for materials should be addressed to T.-H.K. or H.W.Y.

\section{Competing financial interests}

The authors declare no competing financial interests. 


\section{Methods}

Measurements. The STM/STS experiments were performed in an ultrahigh-vacuum $\left(8 \times 10^{-9} \mathrm{~Pa}\right)$ cryogenic STM $(T=78.150 \mathrm{~K})$. The sample temperature was controlled to a precision of at least $\pm 1 \mathrm{mK}$ by means of a temperature controller to minimize unwanted thermal drift during measurements. Sample preparation procedures are described elsewhere ${ }^{23,39}$. All STM images were recorded in the constant-current mode with an electrochemically etched $\mathrm{W}$ tip. The sample bias and tunnelling current were set to $-0.5 \mathrm{~V}$ and $0.1 \mathrm{nA}$, respectively, for STM/STS measurements. STS measurements were performed by recording the $\mathrm{d} I / \mathrm{d} V$ spectra via a lock-in technique with feedback open, using a modulation frequency of approximately $1 \mathrm{kHz}$ at a root-mean-square voltage of $7 \mathrm{mV}$.

Tight-binding calculations. Self-consistent tight-binding calculations were performed with the generalized Su-Schrieffer-Heeger Hamiltonian ${ }^{24}$ to estimate the soliton formation energies and to verify the energetic preference of soliton operations. Soliton states were calculated for a double chain of more than 800 atomic sites per chain with the periodic boundary condition. Soliton state spectra and energy changes were obtained as a function of the distance between two solitons using an adiabatic approach.

Notations for four-fold degenerate ground states. The order parameter $m_{x}$ indicates dimerized orientations leading to two degenerate Peierls-distorted ground states in a single atomic chain (Supplementary Fig. 1a). The order parameter $m_{z}$ denotes the interchain coupling potential term showing how a specific atom (the first atom in the unit cell) on one chain interacts with two atoms on the other chain (Supplementary Fig. 1b). Anti-bonding (Bonding) corresponds to the positive (negative) $m_{z}$. The schematics of four-fold degenerate ground states are shown in Supplementary Fig. 1c. The notations of the ground states from ref. 24 (AA, AB, BA and $\mathrm{BB}$ ) represent only the dimerization orientation of both upper and lower chains, whereas the new notations represent both the dimerization orientation as well as the interchain coupling. Each chain indeed has four-fold ground states: $\mathrm{Aa}, \mathrm{Ab}, \mathrm{Ba}$ and $\mathrm{Bb}$. Four ground states are indicated with $\mathrm{RC}$ solitons in the order-parameter space of $m_{x, \text { upper }}$ and $m_{x, \text { lower }}$ (Supplementary Fig. 1d). Four-fold ground states of upper (upper panel in Supplementary Fig. 1e) and lower chains (lower panel in Supplementary Fig. 1e) with RC solitons are shown in the order-parameter space of each $m_{x}$ and $m_{z}$. Each coloured representation corresponds to each atomic configuration of Supplementary Fig. 1c. As shown in Supplementary Fig. 1e, both chains keep the same chirality, thus one can use the representation of either lower or upper chains alone to represent any ground state of double Peierls-distorted chains.

Electronic states of solitons. STS spectra obtained at the centre of solitons are shown in Supplementary Fig. 2a. RC (LC) solitons have an in-gap state below (above) the Fermi level, whereas AC solitons exhibit in-gap states both below and above the Fermi level. STM images of a RC and a LC soliton (imaging condition: $-0.5 \mathrm{~V}, 0.1 \mathrm{nA}$ ) are shown in Supplementary Fig. 2b,c, respectively. STM height profiles of LC and RC solitons are taken from the middle of the STM images (as indicated by dashed lines in Supplementary Fig. 2b,c), as shown in Supplementary Fig. 2d. It clearly shows that RC solitons have higher apparent STM heights than LC solitons at the centre of solitons due to their electronic states being enhanced from the Fermi level to $-0.5 \mathrm{eV}$.

Chirality of defects. An STM image and a simple schematic diagram of an isolated defect are provided in Supplementary Fig. 3a. In this example, the defect connects between $\mathrm{Aa}$ (red) and $\mathrm{Ba}$ (green) ground states, as highlighted with the thicker arrow in Supplementary Fig. 3b. Such a defect connects two ground states in the same way as LC solitons in the order-parameter space (indicated by arrows in Supplementary Fig. 3b).

Analysis of chirality of trapped solitons. To determine the chirality of solitons, we measured the distances between two defects trapping solitons. STM images of the same five double chains at different times were used as shown in Supplementary Fig. 4a,b. Each number next to the STM images indicates each double chain. White boxes indicate the same chain as Fig. 1h,i showing two defects $\left(D_{1}, D_{2}\right)$ holding a chiral soliton with chiral switching, while black boxes indicate another two defects $\left(\mathrm{D}_{3}, \mathrm{D}_{4}\right)$ holding a LC soliton without chiral switching (see Supplementary Fig. $4 c, d)$. As a result, the distance between $D_{1}$ and $D_{2}\left(D_{3}\right.$ and $\left.D_{4}\right)$ changes from $17 a_{0}\left(7 a_{0}\right)$ to $10 a_{0}\left(11 a_{0}\right)$ with (without) chiral switching. Note that a LC soliton in the black box preserves its chirality for more than $100 \mathrm{~min}$ in Supplementary Fig. 4 .

Correlation between STM apparent heights and possible charge states of defects. To find any correlation between STM apparent heights and possible charge states of defects, we further analysed the same STM images as shown in Fig. 1h,i. STM apparent heights of both defects show a clear dependence on the chirality of solitons in between the defects. When a LC soliton is switched to a RC soliton, both defects [denoted as $D_{1}\left(D_{1}^{*}\right)$ and $D_{2}\left(D_{2}^{*}\right)$ ] become brighter than before chiral switching, as shown in Supplementary Fig. 8a,b. The observed same height change for both defects suggests that their charge states may be modified by almost the same amount. This charge transfer might be responsible for unbalanced charge during chiral switching of charged solitons. Although we cannot precisely determine the initial charge states (denoted as $\alpha, \beta$ ) of defects from this analysis, the reduced size of a positively charged LC soliton due to the attractive Coulomb interaction suggests the initial charge state of the defect $\left(\mathrm{D}_{2}\right)$ should be negative, as described in Supplementary Fig. 8c. Possible chiral switching preserving charge conservation is proposed, as Supplementary Fig. 8d, by considering charge transfer from/to defects.

Random walk analysis of defect displacements. We analysed the random walk behaviour of $D_{2}\left(D_{4}\right)$ with respect to the other defect $D_{1}\left(D_{2}\right)$ shown in Supplementary Fig. 4. In a simple random walk, after $N$ steps, the mean displacements $(\langle x\rangle)$ should be zero, with the exact same probabilities of left- and right-going cases, while the standard deviation of the displacements increases with the square root of $N$. As shown in Supplementary Fig. 9, $\mathrm{D}_{2}$ shows a biased random walk with a slightly higher left-going probability, whereas $\mathrm{D}_{4}$ shows an almost unbiased random walk for small $N(<15)$. However, both cases deviate from a simple random walk for large $N$. This suggests that such defects are bounded due to chiral solitons; thus, they prefer to remain close to each other. Note that red curves are fitted to the simple random walk using data for small $N(<15)$ in Supplementary Fig. 9.

Data availability. The data that support the plots within this paper and other findings of this study are available from the corresponding author upon reasonable request.

\section{References}

39. Yeom, H. W. et al. Instability and charge density wave of metallic quantum chains on a silicon surface. Phys. Rev. Lett. 82, 4898-4901 (1999). 